\title{
LEVANTAMENTO PRELIMINAR DE PTERIDOPHYTA DO PARQUE ESTADUAL DO RIO DOCE (MG)'
}

\author{
Deusângela Graçano \\ Jefferson Prado ${ }^{3}$ \\ Aristéa Alves Azevedo ${ }^{4}$
}

Recebido e Aceito em 27/08/1998

\begin{abstract}
RESUMO - (Levantamento preliminar de Pteridophyta do Parque Estadual do Rio Doce. MG. Brasil). Foi realizado um levantamento preliminar de Pteridophyta no Parque Estadual do Rio Doce, sendo coletadas 15 famílias, 27 gêneros e 57 espécies. As famílias encontradas foram Aspleniaceae. Blechnaceae, Dennstaedtiaceac. Dryopteridaceae, Gleicheniaceae. Hymenophyllaceae. Lomariopsidaceae. Osmundaceac. Polypodiaceae, Pteridaceae. Schizaeaceae, Selaginellaceae, Tectariaceae. Thelypteridaceae e Woodsiaceae. Destas, as mais representativas foram Pteridaceae, com 29,8\% das espécies distribuídas em quatro gêneros (Adiantum, Hemionitis, Pityrogramma e Pteris) e Thelypteridaceae que apresentou 17,5\% das espécies, incluidas em dois gêneros (Macrothelypteris e Thelypteris). As demais famílias variaram de $1.8-8,8 \%$ do total das espécies. Das 57 espécies registradas no Parque, 22 apresentaram distribuição bastante restrita, sendo Pteridaceae e Thelypteridaceae as duas famílias de ocorrência mais ampla. Análises do solo das trilhas do Parque revelaram solo ácido, com $\mathrm{pH}$ variando de 4,3-5,1, preferencialmente argiloso a muito argiloso e com teores médio a baixo de $\mathrm{P}, \mathrm{Ca}, \mathrm{Al}$ e $\mathrm{Mg}$.
\end{abstract}

Palavras-chave - Pteridophyta, Parque Estadual do Rio Doce (MG), flora pteridofítica

ABSTRACT - (Pteridophyta preliminary survey at Rio Doce State Park, MG. Brazil). A Pteridophyta preliminary survey was carried out at Rio Doce State Park, being identified 15 families, 27 genera. and 57 species. The following families were found: Aspleniaceae. Blechnaceae. Dennstaedtiaceae, Dryopteridaceac. Gleicheniaceae, Hymenophyllaceac, Lomariopsidaceac, Osmundaceae, Polypodiaceae. Pteridaceae. Schizaeaceae, Selaginellaceae, Tectariaceac, Thelypteridaceae, and Woodsiaceae. Among them, the most representatives were Pteridaceae, with $29.8 \%$ of the collected species. distributed in four genera (Adiantum. Hemionitis. Pityrogramma, and Pteris) and Thelypteridaceae with $17.5 \%$ of the species included into two genera (Macrothelypteris and Thelypteris). The remaining families ranged from 1.8 to $8.8 \%$ of the species. Out of 57 registered species in the Park. 22 showed a restricted distribution;

\section{Auxilio FAPEMIG}

Bolsista da CAPES (Demanda Social). Curso de Pós-Graduação em Botânica, Departamento de Biologia Vegetal, Universidade Federal de Viçosa (UFV). CEP 36571-00. Viçosa, MG, Brasil

Instituto de Botânica. C. Postal 4005. CEP 01061-970. São Paulo. SP. Brasil

+ Departamento de Biologia Vegetal, UFV. CEP 36571-000. Viçosa, MG, Brasil 
on the other hand Pteridaceae and Thelypteridaceae were the ones with a broader occurrence. Physical and chemical soil analysis of the Park's paths presented acid $\mathrm{pH}(4.3-5.1)$, variable clay proportion and medium to low $\mathrm{P}, \mathrm{Ca}, \mathrm{Al}$ and $\mathrm{Mg}$ content.

Key words - Pteridophyta. Rio Doce State Park (MG). pteridophytic flora

\section{Introdução}

O Parque Estadual do Rio Doce, criado em 1944 e administrado pelo Instituto Estadual de Florestas de Minas Gerais (IEF-MG) desde 1962, é hoje a maior área contínua de Floresta Tropical do Estado de Minas Gerais, possuindo grande variedade de espécies e tendo sido considerado um remanescente de Mata Atlântica (Gilhuis 1986). A classificação que melhor descreve a vegetação do Parque é aquela apresentada por Veloso et al. (1991), caracterizando-o como Floresta Estacional Semidecidual Submontana ou Floresta Tropical Subcaducifólia. Contudo, são poucos os dados disponíveis sobre a vegetação do Vale do Rio Doce que atualmente se apresenta constituída por mosaicos de florestas primárias e secundárias, sendo estas últimas originadas em decorrência de grandes queimadas ocorridas, principalmente, durante os anos 60 (SOCT 1981).

De acordo com Tryon \& Conant (1975), muitos estudos são ainda necessários, principalmente nos trópicos, para que se possa entender aspectos fundamentais das interrelações das Pteridophyta e seus ambientes. Segundo Page (1979), o papel ecológico desse grupo, num amplo espectro de comunidades vegetais, tem sido negligenciado, apesar da diversidade de formas estruturais e da extraordinária variedade de habitats explorados pelas Pteridophyta, desde regiões Árticas e Antárticas até o coração de Florestas Tropicais Úmidas, ao nível do mar ou em altitudes elevadas.

Estudos sobre a influência dos fatores edáficos na distribuição das Pteridophyta (Carlson 1979; Graves \& Monq 1982; Petersen 1985; Ranal 1995) têm corroborado as observações de Wherry (1920) de que a ocorrência de determinadas espécies está mais estreitamente relacionada com a composição mineral do que com as características físicas do solo. Segundo Tuomisto \& Poulsen (1994) a especialização edáfica verificada em muitas espécies de Pteridophyta justifica seu uso como indicadores da fertilidade do solo, devendo ser considerada nos estudos dos padrões de distribuição geográfica das espécies.

Tendo em vista a escassez de dados sobre as Pteridophyta no Brasil, em especial em Minas Gerais, o presente estudo foi realizado com os objetivos de efetuar o levantamento das Pteridophyta ocorrentes no Parque e verificar sua distribuição nas diferentes trilhas; determinar as famílias com maior número de espécies e fornecer dados preliminares sobre o solo das trilhas, para subsidiar estudos sobre a distribuição das espécies e suas relações com o solo.

\section{Material e métodos}

O Parque Estadual do Rio Doce (PERD) fica localizado no Estado de Minas Gerais, nos municípios de Timóteo, Marliéria e Dionísio, e compreende cerca de 36.000 ha, sendo limitado a leste pelo Rio Doce e ao norte pelo Rio Piracicaba (IEF 1994). Está 


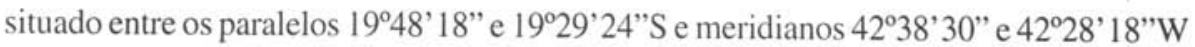
(IEF 1994) (Fig. 1).

Distingue-se, no Parque, um sistema de 38 a 44 lagos de diferentes tamanhos, originados pela paleo-drenagem do Rio Doce, cuja evolução geomorfológica e biológica é bastante peculiar (SOCT 1981). A altitude do Parque varia de $230 \mathrm{~m}$ a $515 \mathrm{~m}$ (IEF 1994) e, de acordo com o PROJETO RADAM (1977, apud CETEC 1982), no relevo predominam encostas originadas pela deposição fluvial.

Segundo Antunes (1986), o Parque está localizado em área de clima de transição entre os tipos Aw e Cwa, na escala de Koeppen (Koeppen 1948), caracterizados por um período de inverno seco e outro de verão chuvoso. De acordo com os dados climáticos mensais obtidos no Posto Meteorológico do viveiro de mudas do PERD, para o período de setembro/ 1995 a dezembro/ 1996 , a temperatura média máxima foi de $34^{\circ} \mathrm{C}$ em fevereiro e a média mínima, de $13^{\circ} \mathrm{C}$ em julho. A precipitação anual foi de $1.131 \mathrm{~mm}$, com máxima de $685 \mathrm{~mm}$ em dezembro e mínima de $2 \mathrm{~mm}$ em junho. A umidade relativa média do ar durante o ano de estudo foi de $86 \%$.

Foram realizadas 17 excursões para coleta de material botânico dos representantes da divisão Pteridophyta, ocorrentes no Parque Estadual do Rio Doce, no período de setembro de 1995 a setembro de 1996. As coletas foram realizadas ao longo das trilhas e estradas já demarcadas no Parque (Fig. 1). As trilhas Vinhático, Campolina, Mombaça, Salão Dourado e Ilha do Clei são de interior de mata, sendo a do Vinhático a mais visitada por serem realizados, neste local, cursos de campo e aulas de Educação Ambiental. Aníbal, Porto Capim e Lagoa Carioca são estradas estreitas, de acesso somente aos funcionários do Parque. Nas trilhas do Aníbal e Porto Capim as coletas foram realizadas principalmente nos barrancos sombreados, à margem da estrada, enquanto na estrada de acesso à Lagoa Carioca as coletas foram feitas na margem da estrada, em local aberto e mais ensolarado. As estradas de acesso ao Restaurante e à Ponte Queimada são largas e com tráfego constante de veículos, sendo as coletas realizadas nos barrancos. As espécies epífitas foram coletadas ora no interior de mata, ora na margem das estradas, sobre troncos caídos ou árvores.

Todo material botânico coletado foi prensado no campo, herborizado seguindo as técnicas usuais e incluído no acervo do Herbário (VIC) do Departamento de Biologia Vegetal da Universidade Federal de Viçosa. A identificação do material botânico foi realizada com auxílio de literatura especializada, por meio de consultas a especialistas e por comparação com material de herbário já identificado. Duplicatas foram doadas para o Herbário do Instituto de Botânica de São Paulo (SP), Herbário BHCB da Universidade Federal de Minas Gerais e Herbário do Parque Estadual do Rio Doce (PERD).

A apresentação da listagem para famílias, gêneros e espécies seguiu o proposto por Moran \& Yatskievych (1995). As abreviações dos nomes dos autores dos nomes científicos seguiram Pichi-Sermolli (1996).

No campo, foram feitas anotações referentes ao hábito e habitat de cada exemplar. $\mathrm{Na}$ Ilha do Clei, devido à dificuldade de acesso à região norte do Parque, foi realizada uma única coleta de material botânico e não foi feita amostragem do solo da trilha.

Para análise física e química do solo das trilhas, foram coletadas amostras compostas, com cerca de $300 \mathrm{~g}$ cada, em 18 locais distintos, utilizando-se para isso um 
trado tipo Holandês. Em cada local foram feitas coletas em diferentes pontos, numa área de aproximadamente $1 \mathrm{~m}^{2}$, às profundidades de 0-5 e 5-10 cm. Foram amostrados 11 locais no interior da mata (três na Campolina, três na Mombaça, um no Salão Dourado,

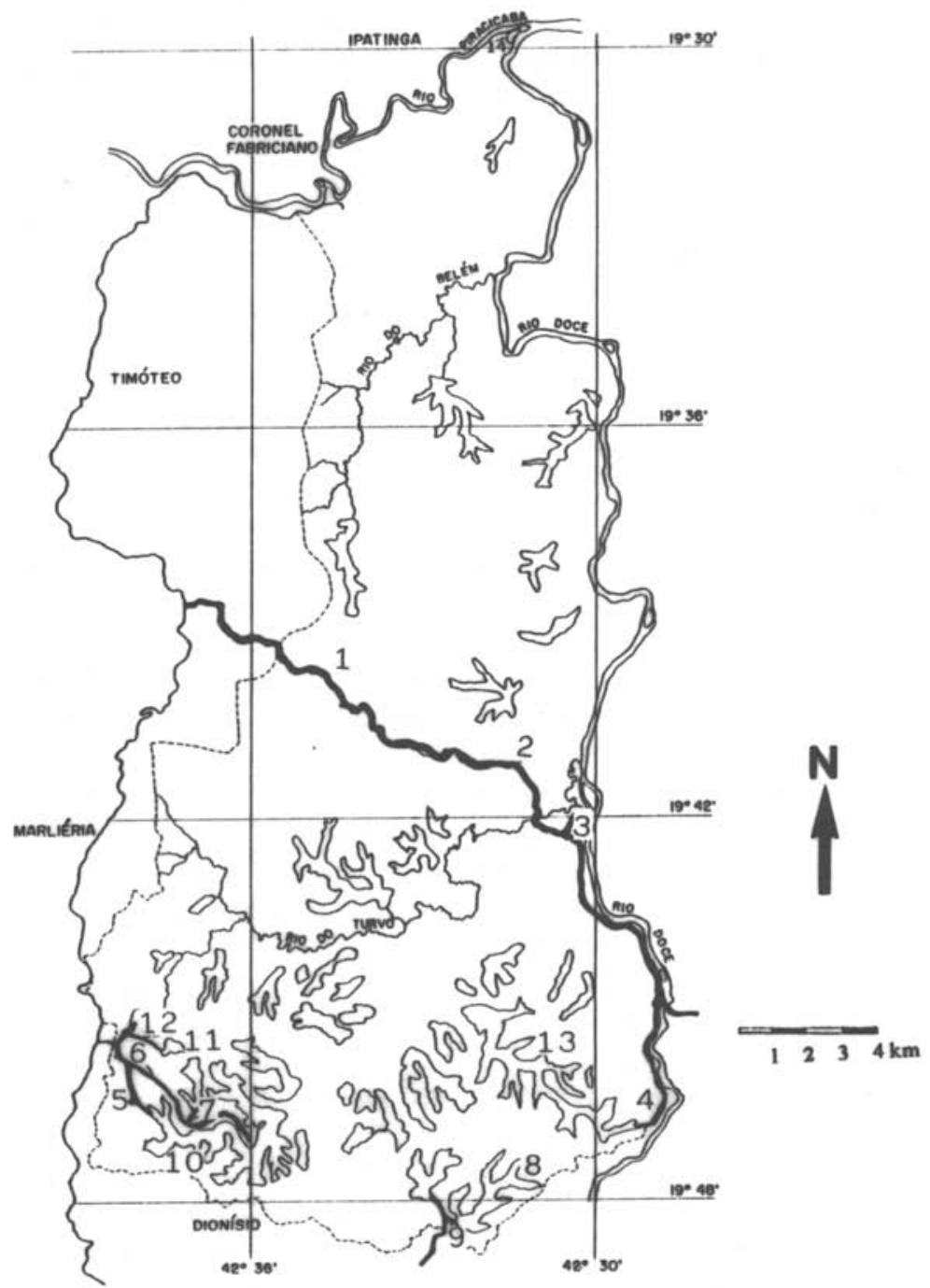

Figura 1. Mapa do Parque Estadual do Rio Doce - MG, limitado ao norte pelo rio Piracicaba, a leste pelo rio Doce, à oeste e sul pela linha tracejada. Trilhas e estradas onde foram realizadas as coletas e principais lagoas do Parque: 1- Trilha Salão Dourado; 2- Estrada da Ponte Queimada; 3 - Trilha da Campolina; 4- Trilha do Aníbal; 5- Trilha Porto Capim; 6- Estrada do Restaurante; 7- Trilha do Vinhático; 8- Lagoa dos Patos; 9- Trilha da Mombaça; 10- Lagoa Dom Helvécio; 11- Lagoa Carioca; 12- Estrada da Lagoa Carioca; 13- Lagoa do Aníbal; 14- Ilha do Clei. Fonte: Carta imagem de satélite: escala $1: 200.000$. 
quatro no Vinhático), seis em região de barranco (três no Anibal, três no Porto Capim) e um na estrada de acesso, bem próximo à Lagoa Carioca.

As amostras de solo foram enviadas ao Laboratório de Física e Química do Departamento de Solos da Universidade Federal de Viçosa (UFV), onde foram realizadas análises de textura pelo método da pipeta (EMBRAPA 1979) e análise química (Defelipo \& Ribeiro 1991).

\section{Resultados}

No levantamento preliminar das Pteridophyta do Parque Estadual do Rio Doce, foram encontradas 15 famílias, 27 gêneros e 57 espécies (Tab. 1), distribuídas nas diferentes trilhas (Tab. 2). Do total de espécies, 29,8\% pertencem à família Pteridaceae, $17,5 \%$ à família Thelypteridaceae, $8,8 \%$ às famílias Schizaeaceae e Tectariaceae, e 1,8$7,0 \%$ às demais famílias (Fig. 2). Das 15 famílias coletadas 33\% estão representadas por apenas uma espécie (Tab. 1).

Foram registradas 17 espécies de Pteridaceae distribuídas em quatro gêneros, sendo Adiantum o mais representativo, com 12 dessas espécies, e 10 espécies de Thelypteridaceae, das quais nove pertencem ao gênero Thelypteris (Tab. 2).

Thelypteridaceae foi a família de ocorrência mais ampla, sendo coletada em nove das dez trilhas analisadas, seguida por Pteridaceae e Schizaeaceae, que ocorreram em oito trilhas (Tab. 2 ; Fig. 3). Pteridaceae não foi encontrada na Estrada da Ponte Queimada e Estrada do Restaurante (Fig. 3). Nestas trilhas, entretanto, foram coletados representantes de Schizaeaceae, família não registrada na Ilha do Clei e Salão Dourado.

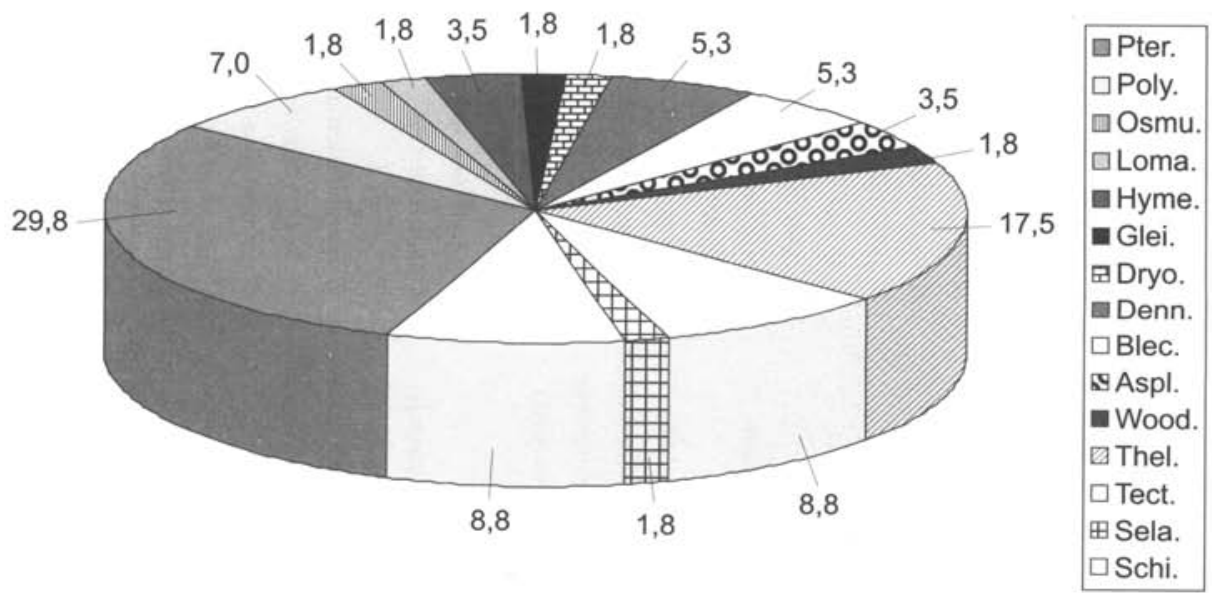

Figura 2. Distribuição percentual de espécies de Pteridophyta, por família, no Parque Estadual do Rio Doce, MG. Aspl.-Aspleniaceae, Blec.-Blechnaceae, Denn.-Dennstaedtiaceae, Dryo.-Dryopteridaceae, Glei.-Gleicheniaceae, Hyme.-Hymenophyllaceae, Loma.-Lomariopsidaceae, Osmu.-Osmundaceae, Poly.-Polypodiaceae, Pter.-Pteridaceae, Schi.-Schizaeaceae, Sela.-Selaginellaceae, Tect.-Tectariaceae, Thel.-Thelypteridaceae, Wood.-Woodsiaceae. 


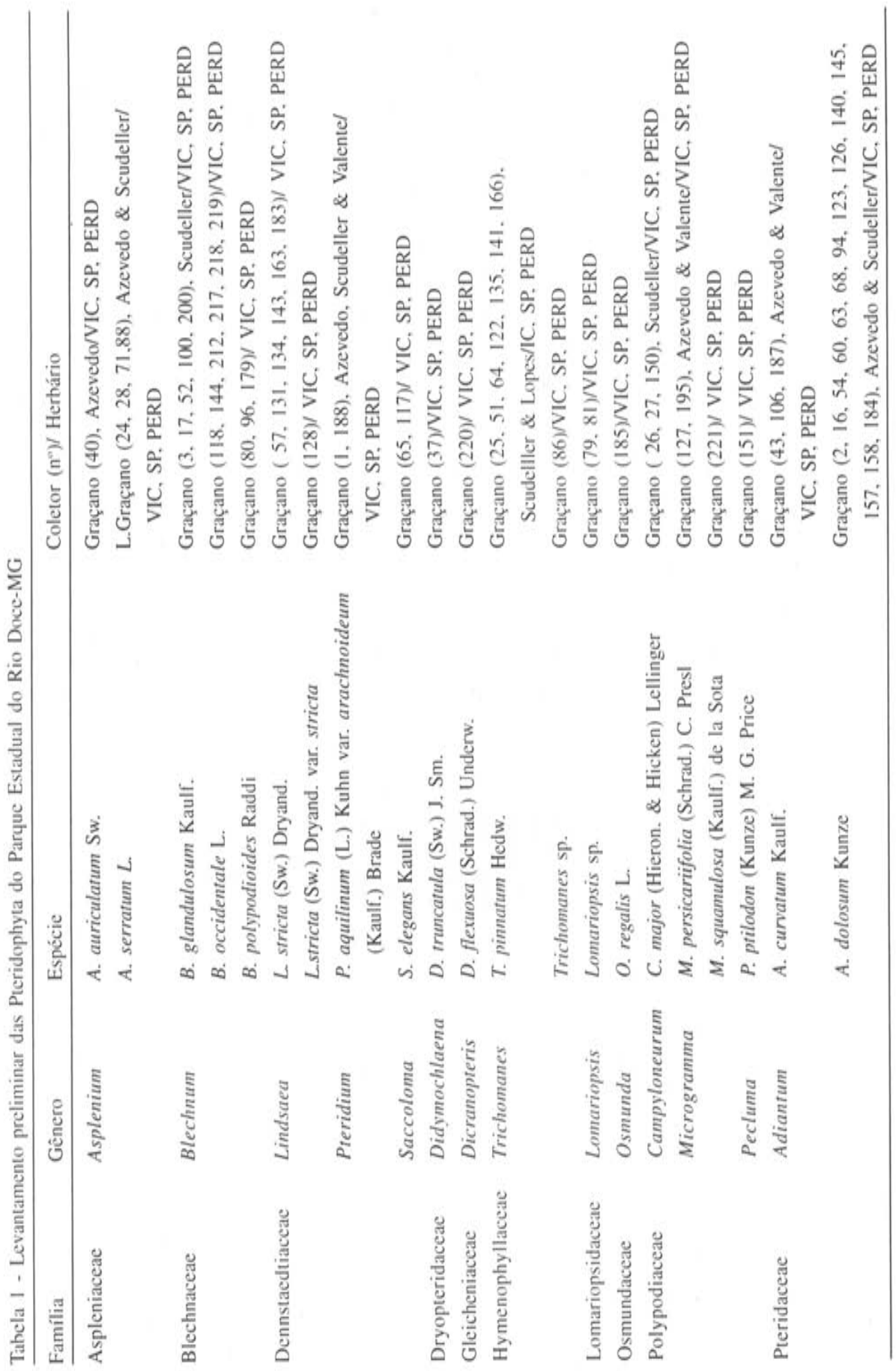




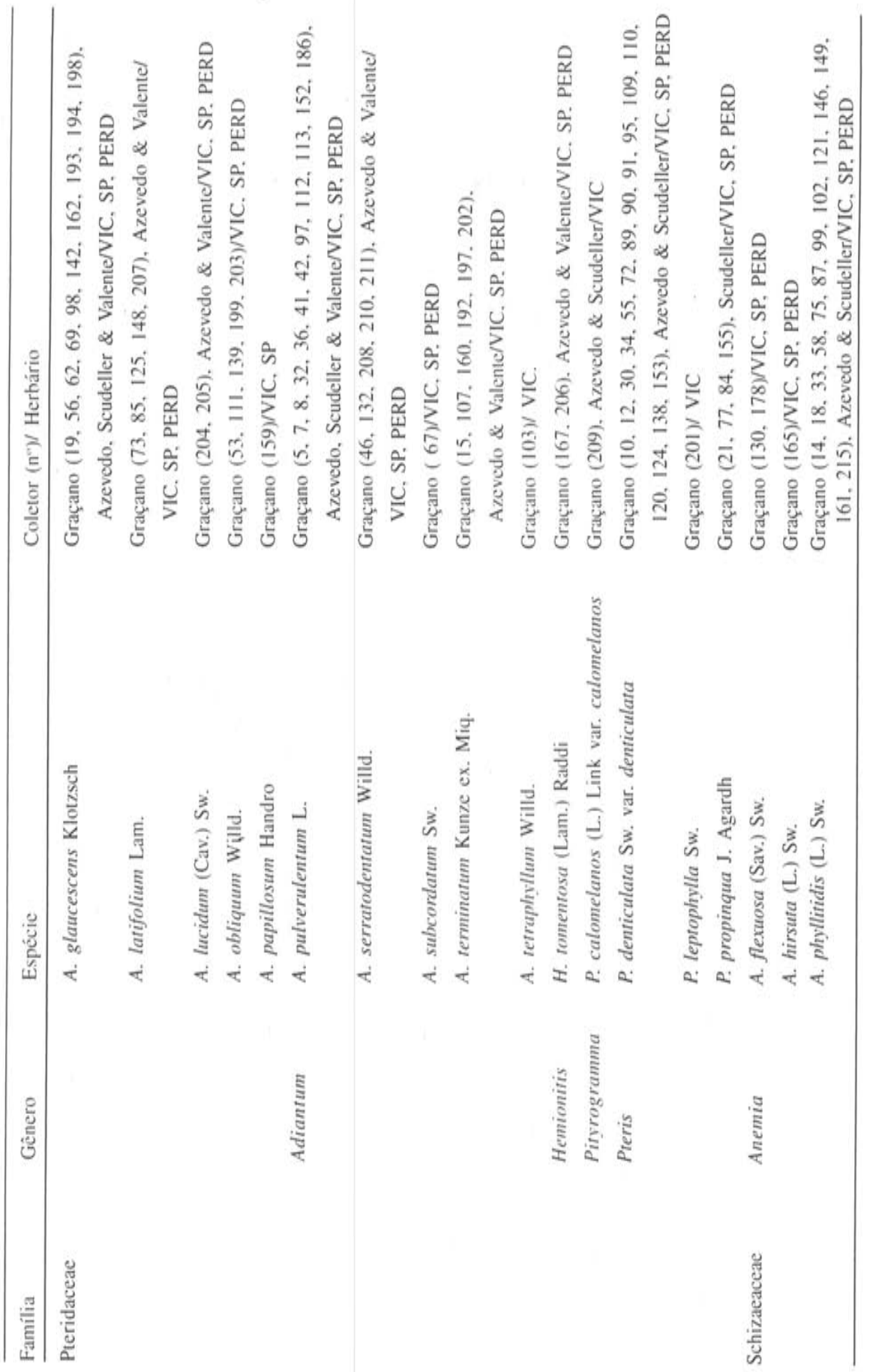




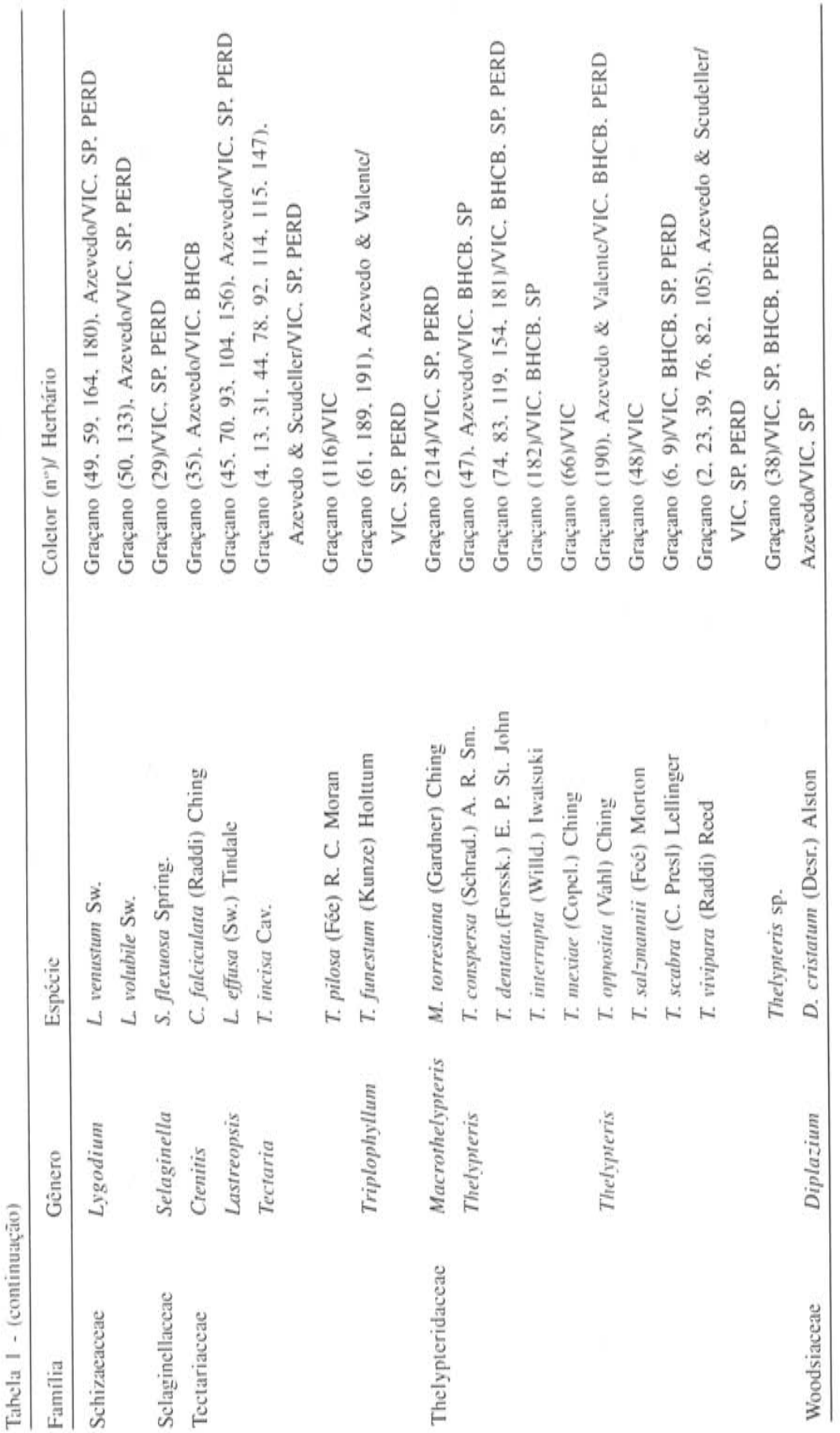




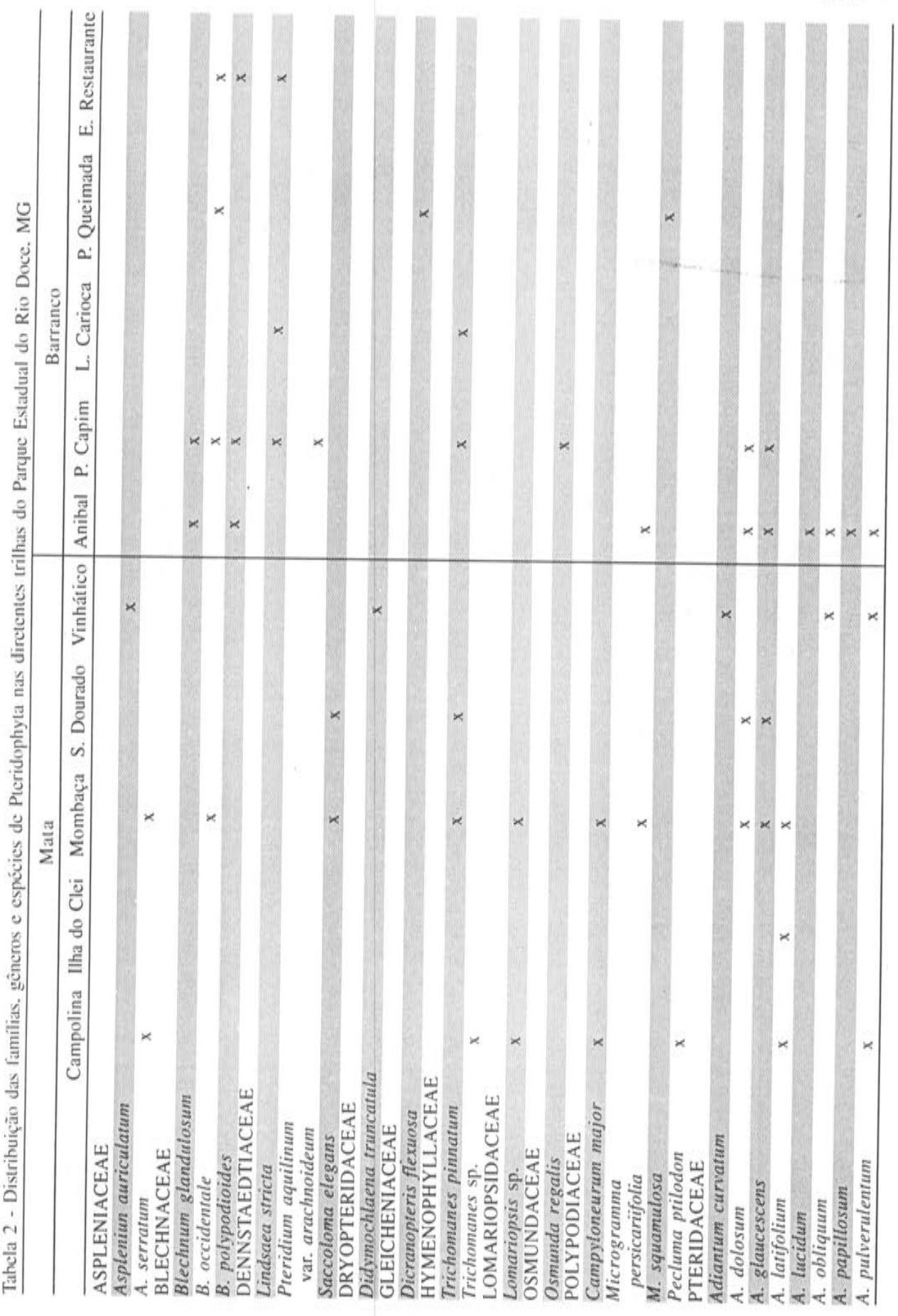




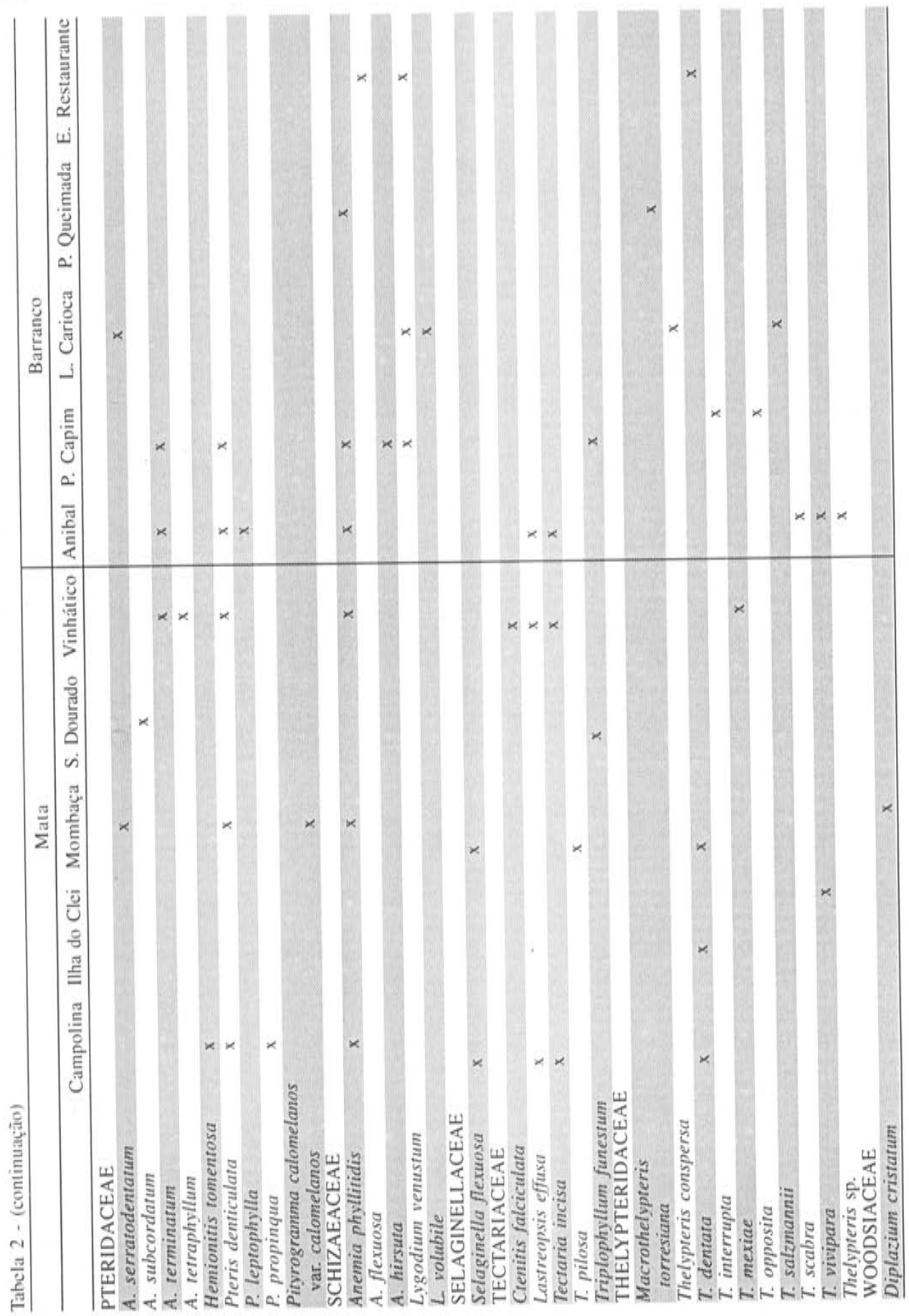


Thelypteridaceae foi a família de ocorrência mais ampla, sendo coletada em nove das dez trilhas analisadas, seguida por Pteridaceae e Schizaeaceae, que ocorreram em oito trilhas (Tab. 2 ; Fig. 3). Pteridaceae não foi encontrada na Estrada da Ponte Queimada e Estrada do Restaurante (Fig. 3). Nestas trilhas, entretanto, foram coletados representantes de Schizaeaceae, família não registrada na Ilha do Clei e Salão Dourado. Outras famílias, como Dryopteridaceae, Gleicheniaceae, Osmundaceae e Woodsiaceae estão, até o presente momento, restritas a uma única trilha, Vinhático, Ponte Queimada, Porto Capim e Ilha do Clei, respectivamente (Tab. 2; Fig. 3).

Anemia phyllitidis (Schizaeaceae) e Pteris denticulata var. denticulata (Pteridaceae) foram as espécies que ocorreram em maior número de trilhas, seis e cinco respectivamente (Tab. 2). Das 57 espécies registradas, 22 apresentaram distribuição bastante restrita, ocorrendo em apenas uma trilha.

As trilhas da Mombaça e Porto Capim apresentaram maior diversidade em espécies; entretanto na Mombaça ocorre maior número de famílias do que no Porto Capim (Tab. 2; Fig. 3). Comparando-se as trilhas do interior da mata com maior número de espécies (Campolina, Mombaça e Vinhático), observa-se menor diversidade de famílias na trilha do Vinhático (Tab. 2; Fig. 3).

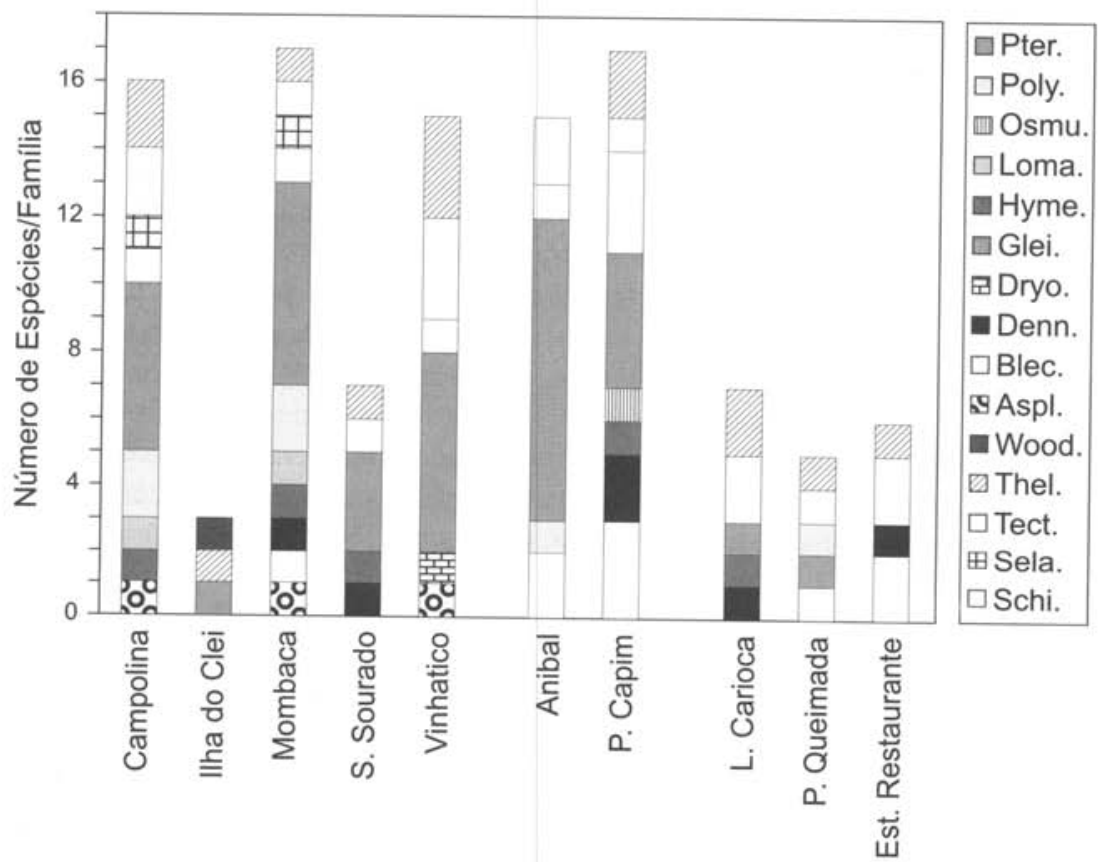

Figura 3. Número de espécies de Pteridophyta, por família, ocorrentes nas diferentes trilhas do Parque Estadual do Rio Doce, MG. Aspl.-Aspleniaceae, Blec.-Blechnaceae, Denn.-Dennstaedtiaceae, Dryo.Dryopteridaceae, Glei.-Gleicheniaceae, Hyme.-Hymenophyllaceae, Loma.-Lomariopsidaceae, Osmu.Osmundaceae, Poly.-Polypodiaceae, Pter.-Pteridaceae, Schi.-Schizaeaceae, Sela.-Selaginellaceae, Tect.Tectariaceae, Thel.-Thelypteridaceae, Wood.-Woodsiaceae. 
As espécies epífitas Asplenium serratum (Aspleniaceae), Trichomanes sp. (Hymenophyllaceae), Lomariopsis sp. (Lomariopsidaceae), Campyloneurum major, Microgramma persicariifolia, M. squamulosá, Pecluma ptilodon (Polypodiaceae) e Hemionitis tomentosa (Pteridaceae) foram, na maioria das vezes, coletadas nas trilhas da Campolina e Mombaça.

Os resultados obtidos na análise química do solo revelaram que o solo das trilhas é ácido, com pH variando de 3,9-5,1 (Tab. 3). Na maioria das trilhas, a quantidade de matéria orgânica, medida pelo teor de carbono, é maior na profundidade $0-5 \mathrm{~cm}$, do que na profundidade de $5-10 \mathrm{~cm}$. A concentração de alumínio é baixa na maioria das trilhas; nas trilhas Aníbal, Salão Dourado e Mombaça foram observados os valores mais altos. Apenas nos locais onde foram registrados os valores mais elevados de alumínio foram coletadas as espécies Adiantum papilosum e A. subcordatum, respectivamente, trilha do Aníbal (ponto 10) e trilha Salão Dourado (ponto 14). Os solos de barrancos situados à margem de estradas, quando comparados com os de mata, apresentaram quantidade mais baixa de carbono, fósforo e cálcio, além de menor capacidade de troca catiônica efetiva (Tab. 3).

A análise granulométrica mostrou que predominam solos argilosos a muito argilosos nos pontos amostrados (Tab. 3). Verificou-se maior variação na textura do solo na trilha do Anibal. Na estrada de acesso à Lagoa Carioca a granulometria indicou solo franco-argilo-arenoso; no ponto amostrado ocorre apenas Adiantum serratodentatum.

\section{Discussão e conclusões}

Todas as espécies de Pteridophyta encontradas, até o momento, mostraram distribuição desigual nas diferentes trilhas e estradas do Parque Estadual do Rio Doce. Dentre os fatores do ambiente que influenciam na distribuição das espécies, as condições físicas e químicas do solo, a irradiância, a temperatura e a umidade do ar e do solo são relevantes (Tuomisto \& Ruokolainem 1994). Segundo Ranal (1995), a distribuição das Pteridophyta terrestres deve estar relacionada com o teor de nutrientes e pH do solo. $\mathrm{O}$ fogo, como salientado por Carvalho (1982), também pode influenciar na distribuição e frequiência das espécies de Pteridophyta. Como no Parque ocorreu grande incêndio em 1967, atingindo vários pontos de diversas trilhas, e pequenos focos, em 1993, esse é um fator a ser considerado.

Barrancos situados em ambientes de mata (Porto Capim e Aníbal) apresentaram número de espécies e diversidade de famílias comparável às trilhas de mata, mas em barrancos à margem de estradas o número de espécies foi reduzido. Segundo Page (1979), estes habitats construídos pelo homem, apesar de artificiais, são comparáveis aos formados pela erosão natural, onde a colonização por Pteridophyta é rápida, contribuindo para a estabilidade do habitat; nestes locais estabelecem-se fetos de pequeno porte, com frondes coriáceas, com rápido desenvolvimento vegetativo e maturidade precoce, sendo característicos destes ambientes táxons de ampla distribuição geográfica, como Blechnum occidentate, Pityrogramma calomelanos, entre outros.

Os dados sobre o solo das trilhas revelaram que o solo do Parque apresenta, de acordo com a Comissão de Fertilidade do Solo do Estado de Minas Gerais (1989), baixo 


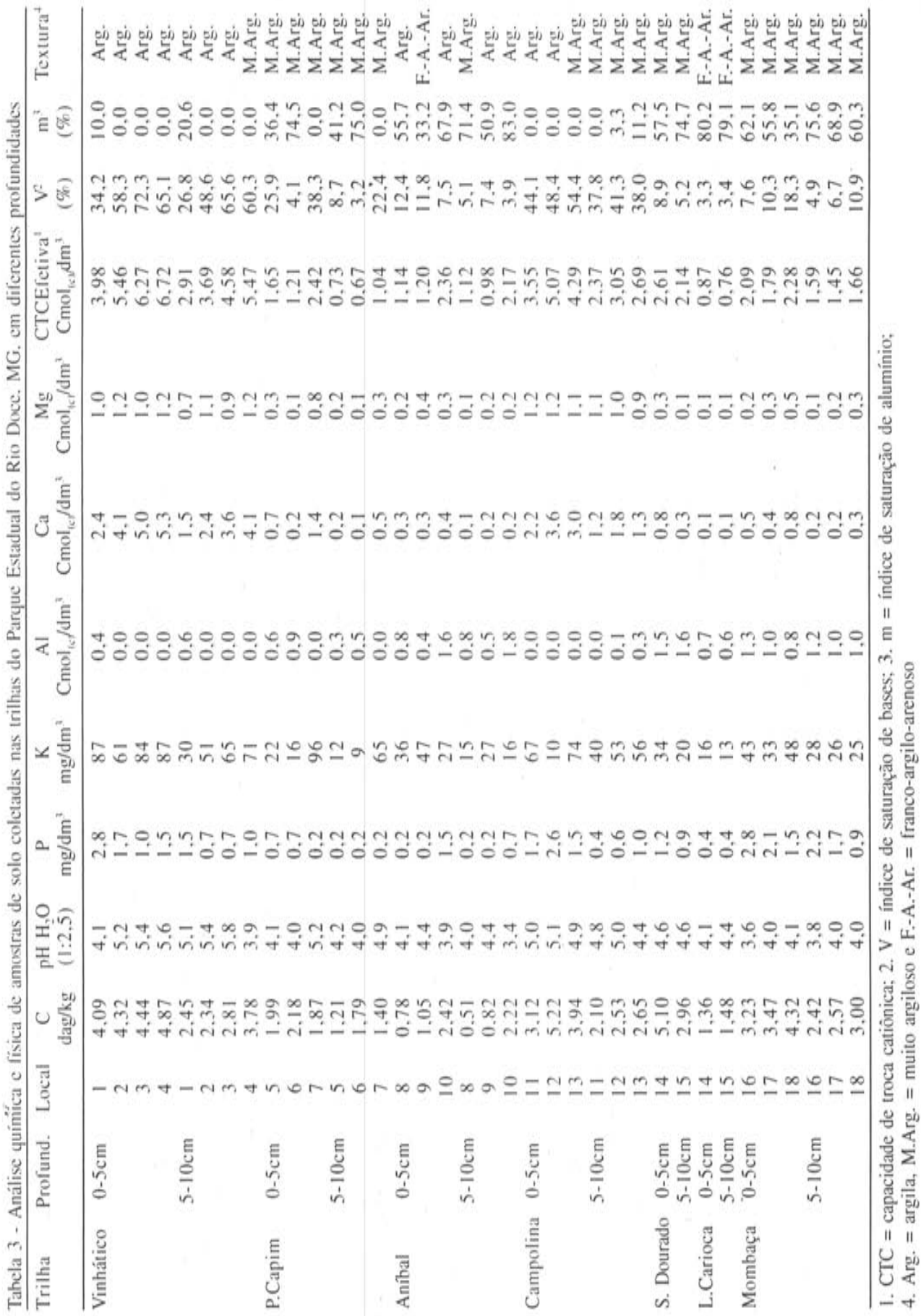


a médio teor de fósforo, potássio, cálcio, magnésio e alumínio. De maneira geral, todas as amostras representam solos que possuem pelo menos um fator limitante para boa fertilidade. Os fatores limitantes mais comuns foram o teor de fósforo e a acidez. Estudos realizados pelo SOCT (1981) indicam que em $67,92 \%$ da área total do.Parque predominam solos que apresentam horizonte $\underline{B}$ textural e $\underline{B}$ latossólico, de textura argilosa, álicos e distróficos, com acidez variando de média a elevada.

As amostras oriundas de Campolina e Vinhático podem ser classificadas como aquelas que representaram solos mais férteis. $\mathrm{O}$ valor do índice de saturação de bases (V\%) maior que 50\%, o teor de carbono e a baixa acidez confirmam esta avaliação. No entanto, o nível de fósforo disponível pode ser considerado como limitante. Apesar dos solos da Campolina e Vinhático apresentarem maior fertilidade, a diversidade de famílias no Vinhático foi menor, provavelmente por ser esta trilha sujeita a ação antrópica mais intensa, enquanto em Campolina ocorre mata primária com ação antrópica reduzida. Outro fator a ser considerado, para a trilha do Vinhático, foi a ocorrência de fogo registrado nas décadas de 60 e 90 . A influência do fogo na vegetação, ocasionando redução no número de espécies, foi observada por Gentilini et al. (1981) no estudo fitossociológico realizado no Parque, comparando áreas de floresta primária com áreas alteradas pelo fogo. A vegetação do Parque é hoje um mosaico de formações vegetais em diferentes estádios de sucessão (Andrade et cl. 1997).

Correlacionando a distribuição das espécies de Pteridaceae com as características do solo, pode-se perceber que as amostras mais distróficas são aquelas onde foram encontrados apenas exemplares de uma única espécie, como por exemplo Adiantum papillosum, A. subcordatum e A. serratodentatum. A. papillosum e A. subcordatum são espécies morfologicamente semelhantes, sendo identificada apenas uma população de cada no Parque. A análise do solo nos pontos onde estas espécies foram coletadas evidenciou o mais alto teor de alumínio registrado dentre todas as amostras analisadas. Como não foi analisado o teor de alumínio nos tecidos dessas espécies, serão necessários estudos mais detalhados para esclarecer possível correlação entre a presença de $A$. papillosum e A. subcordatum e o teor de alumínio no solo, bem como verificar a ocorrência de acúmulo de alumínio em seus tecidos. Segundo Ranal (1995), algumas espécies de Pteridophyta são acumuladoras de alumínio, como Polypodium latipes, $P$. pleopeltifolium è Pteris denticulata.

Conforme os dados obtidos no Parque, a maioria das espécies ocorreu em solo argiloso ou muito argiloso. Adiantum serratodentatum foi a única espécie observada em solo franco-argilo-arenoso, próximo à Lagoa Carioca, em clareiras e associada a gramíneas, a Lindsaea stricta e a Melastomataceae. Entretanto, Adiantum serratodentatum também ocorreu em local aberto, próximo à Lagoa dos Patos (Trilha Mombaça), onde o solo é muito argiloso e apresenta constituição química diferente, com teores mais elevados de carbono, fósforo, potássio, cálcio e maior índice de saturação de bases sendo, portanto, mais fértil, quando comparado com o solo do ponto próximo à Lagoa da Carioca. Como Adiantum serratodentatum foi coletado em locais ensolarados, próximos às margens de lagoas, cujos solos apresentam características bastante diversas, pode-se supor que a distribuição dessa espécie deva estar mais relacionada com o fator intensidade luminosa do que com as características do solo. O mesmo foi observado 
por Pereira-Noronha (1989) estudando cinco espécies de Pteridophyta, em matas das bacias do Rio Tietê e Rio Grande, SP.

A maioria das espécies de hábito epifítico (Asplenium serratum, Campyloneurum major, Hemionitis tomentosa, Lomariopsis sp. e Pecluma ptilodon) ocorreu na trilha da Campolina (Tab. 2), talvez pelas condições desta trilha que é considerada fragmento de mata primária. É importante ressaltar o escasso número de Pteridophyta com hábito epifítico na área do Parque. Segundo Fontoura et al. (1997), áreas de mata secundária apresentam reduzido número de epífitas em relação às áreas de mata primária. Neste caso, a escassez de espécies epífitas pode indicar o caráter secundário das matas do PERD.

Como a área do Parque foi considerada remanescente de Mata Atlântica (Gilhuis 1986), cabe uma comparação com estudos realizados em áreas de outros Estados, onde este tipo de mata ocorre, como é o caso da Serra de Macaé de Cima, no Estado do Rio de Janeiro (Sylvestre 1997). Nesta área foram amostradas 80 espécies, sendo Dryopteridaceae (14 spp.), Polypodiaceae (14 spp.), Cyatheaceae (8 spp.) e Lycopodiaceae ( $8 \mathrm{spp}$.) as famílias mais representativas. Deve-se salientar que, enquanto no Parque, Pteridaceae é a família melhor representada, com 17 espécies, na Reserva de Macaé de Cima ocorrem apenas três espécies desta família, as quais não foram registradas no Parque. Assim, as duas regiões são bastante diferentes no que se refere a flora pteridofítica.

Scudeller (1997), realizando um levantamento florístico da família Bignoniaceae, também verificou que as espécies encontradas na área do Parque apresentaram baixa similaridade específica com regiões de Mata Atlântica.

A diversidade de famílias de Pteridophyta ocorrente nos diferentes ambientes do Parque evidencia que o grupo está bem representado não só em duas categorias de vegetação, como estabelecido por Gilhuis (1986). Este autor reconhece dez categorias de vegetação para o PERD: mata alta primária com epífitas, mata alta, mata média a alta com bambuzóides e graminóides, mata média secundária com bambuzóides e graminóides, mata baixa secundária, arvoredo baixo, campo sujo, samambaial, taboal e vegetação de hidrófitas, destacando a presença de pteridófitas na mata alta primária com epífitas vasculares e no samambaial. Desta forma, fica evidenciada a necessidade de estudos intensivos na área do Parque para melhor caracterização de sua flora e correlação da distribuição das espécies com os fatores ambientais.

\section{Agradecimentos}

Ao Instituto Estadual de Florestas, MG, pela infra-estrutura e facilidades proporcionadas durante os trabalhos de campo no Parque Estadual do Rio Doce. Ao Prof. Alexandre Salino, da Universidade Federal de Minas Gerais, pela identificação das Thelypteridaceae. À FAPEMIG, pelo apoio financeiro. Aos assessores, pelas sugestões que contribuíram para o aprimoramento do texto.

\section{Referências bibliográficas}

Andrade, P. M.; Pereira, M. C. A. \& Silva, L. V. C. 1977. The vegetation of Rio Doce State Park. Pp. 15-21. In: Tundisi J. \& Saito Y. (eds.) Limnological studies on the Rio Doce Valley Lakes, Brazil. Brazilian Academy of Sciences. São Paulo University. School of Engineering. São Carlos. 
Antunes, F. Z. 1986. Caracterização climática do Estado de Minas Gerais. Informe Agropecuário 12(138): 9-13.

Carlson. T. J. 1979. The comparative ecology and frequencies of interspecific hybridization of Michigan wood ferns. Michigan Botany 18: 47-56.

Carvalho, I. R. 1982. O gênero Anemia Sw. nos campos rupestres da Cadeia do Espinhaço no Estado de Minas Gerais. UNESP. Rio Claro. Dissertação de Mestrado.

CETEC - Fundação Centro Tecnológico de Minas Gerais. 1982. Levantamento ecológico da vegetação do Parque Florestal Estadual do Rio Doce. Belo Horizonte.

Comissão de Fertilidade do Solo do Estado de Minas Gerais. 1989. Recomendações para o uso de corretivos e fertilizantes em Minas Gerais. $4^{2}$ aproximação. Lavras.

Defelipo, B. V. \& Ribeiro. A. C. 1991. Análise química do solo (metodologia). Viçosa, MG. Universidade Federal de Viçosa, Imprensa Universitária. Boletim de Extensão 29.

EMBRAPA - Empresa Brasileira de Pesquisa Agropecuária. 1979. Manual de métodos de análise de solo. Serviço Nacional de Levantamento e Conservação de Solos, Ministério da Agricultura. Rio de Janeiro.

Fontoura, T.; Sylvestre, L. S.; Vaz, A. M. S. \& Vieira, C. M. 1997. Epifitas vasculares, hemiepifitas e hemiparasitas da Reserva Ecológica de Macaé de Cima. Pp. 89-101. In: Lima H. C. \& GuedesBruni R. R. (eds.) Serra de Macaé de Cima: diversidade florística e conservação em Mata Atlântica. Jardim Botânico do Rio de Janeiro. Rio de Janeiro.

Gentilini, E.; Carvalho, I. R. \& Teixeira, M. C. B. 1981. Vegetação do Parque Estadual do Rio Doce. In: CETEC - Fundação Centro Tecnológico de Minas Gerais. Programa de pesquisas ecológicas do Parque Estadual do Rio Doce. Vol. 2. Belo Horizonte.

Gilhuis, J. P. 1986. Vegetation survey of the Parque Florestal Estadual do Rio Doce, MG, Brazil. Universidade Federal de Viçosa. Viçosa. Instituto Estadual de Florestas, Agricultural University of Wagenengen. Dissertação de Mestrado.

Graves, J. H. \& Monk, C. D. 1982. Herb-soil relationships on a lower north slope over marble. Bulletin Torrey Botanical Club 109: 500-507.

IEF - Instituto Estadual de Florestas. 1994. Pesquisas prioritárias para o Parque Estadual do Rio Doce, Brasil. Belo Horizonte.

Koeppen, W. 1948. Climatologia: com un studio de los climas de la tierra. Tradução de. P.R. Hendrichs Pérez. Fondo de Cultura Economica. México. p. 152-182.

Moran, R. C. \& Yatskievych. G. 1995. Pteridaceae: Adiantaceae, Parkeriaceae, descripción de la família y clave genérica. Pp. 104-117. In: Davidse G.; Sousa M. S. \& Knapp S. (eds.) Flora Mesoamericana. Vol. I. Psilotaceae-Salviniaceae. Ciudade Universitária, Universidad Nacional Autónoma de México. México, D.F.

Page, C. N. 1979. The diversity of ferns: an ecological perspective. Pp. 9-56. In: Dyer A. F. (ed.) The experimental biology of ferns. Academic Press. London.

Pereira-Noronha, M. R. 1989. Formas de vida e reprodução em pteridófitas. UNESP. Rio Claro. Tese de Doutorado.

Petersen, R. L. 1985. Towards an appreciation of fern edaphic niche requiirements Pp. 93-103. In: Dyer A. F. \& Page C. N. (eds.) Biology of pteridophytes. The Royal Society of Edinburgh. Edinburgh.

Pichi-Sermolli, R. E. G. 1996. Authors of scientific names in Pteridophyta. Royal Botanic Gardens. Kew.

Ranal, M. A. 1995. Estabelecimento de pteridófitas em mata semidecídua do Estado de São Paulo. 2. Natureza dos substratos. Revista Brasileira de Biologia 55(4): 583-594.

Scudeller, V. V. 1997. A tribo Bignoniae Spreng. (Bignoniaceae) no Parque Estadual do Rio Doce-MG. Universidade Federal de Viçosa. Viçosa. Dissertação de Mestrado.

SOCT - Sistema Operacional de Ciência e Tecnologia. 1981. Programa de pesquisas ecológicas no Parque Florestal Estadual do Rio Doce. Vol. 2. CETEC. Belo Horizonte.

Sylvestre, L. S. 1997. Pteridófitas da Reserva Ecológica de Macaé de Cima. Pp. 41-52. In: Lima H. C. \& Guedes-Bruni R. R. (eds.) Serra de Macaé de Cima: diversidade florística e conservação em Mata Atlântica. Jardim Botânico do Rio de Janeiro. Rio de Janeiro.

Tryon, R. M. \& Conant, D. S. 1975. The ferns of Brazilian Amazonia. Acta Amazonica 5(1): 2334. 
Tuomisto, H. \& Poulsen, A. D. 1994. Influence of edaphic specialization on pteridophyte distribution in neotropical rain forests. Pp. 1-19. In: Tuomisto $\mathrm{H}$. Ecological variation in the rain forests of peruvian amazonia: integrating fern distribution patterns with satellite imagery. University of Turku. Reports from the Department of Biology $n^{\circ} 45$. Turku.

Tuomisto, H. \& Ruokolainen. K. 1994. Distribution of Pteridophyte and Melastomataceae along an edaphic gradient in a Amazonian rain forest. Pp. 25-34. In: Tuomisto H. Ecological variation in the rain forests of peruvian amazonia: integrating fern distribution patterns with satellite imagery. University of Turku. Reports from the Department of Biology $n^{\circ} 45$. Turku.

Veloso, H. P.; Rangel Filho, A. L. R. \& Lima, J. C. A. 1991. Classificação da vegetação brasileira, adaptada a um sistema universal. IBGE. Rio de Janeiro.

Wherry, E. T. 1920. The soil reactions of certain rock ferns-I. II. American Fern Journal 10: 1522. $45-52$. 